\title{
Influencia de los materiales plásticos sobre las características de los engobes. I Tipo de material arcilloso
}

\author{
A. MORENO, E. BOU, Ma C. NAVARRO, J. GARCÍA \\ Instituto de Tecnología Cerámica. Asociación de Investigación de las Industrias Cerámicas. \\ Universitat Jaume I. Castellón. España.
}

\begin{abstract}
Uno de los componentes principales de los engobes cerámicos es el material arcilloso, el cual confiere las propiedades adecuadas para llevar a cabo la correcta aplicación de la suspensión de engobe y permitir la obtención de una capa cruda con la apropiada resistencia mecánica y adherencia al soporte.

En el presente trabajo se han realizado una serie de ensayos con el objeto de determinar cómo afecta el tipo y porcentaje de material arcilloso a las propiedades de las suspensiones de engobe (comportamiento reológico) y de las capas crudas obtenidas a partir de ellas (compacidad, adherencia y tiempo de secado superficial). Los materiales arcillosos ensayados han sido los utilizados habitualmente en la preparación de los engobes: arcilla blanca, caolín y bentonita.

El análisis de los resultados obtenidos permite concluir que todas las características del engobe tenidas en cuenta varían considerablemente al modificar el tipo de material arcilloso. Se ha podido comprobar que dichas variaciones son debidas a cambios en el empaquetamiento de la capa cruda y en la plasticidad del engobe, que se producen al modificar el tipo de material arcilloso.
\end{abstract}

Palabras clave: engobe, materiales arcillosos, comportamiento reológico, propiedades de la capa cruda

Effect of plastic materials on engobe characteristics. I. Type of clayey material.

One of the major ceramic engobe constituents is the clayey material. This material contributes suitable properties to the engobe suspension for correct application and yields an unfired layer with adequate mechanical strength and bonding to the body.

A series of tests was conducted in the present study to determine how the type and proportion of clayey material affected engobe suspension properties (rheological behaviour) and resulting unfired engobe layer properties (compactness, bonding and surface drying time). The tested clayey materials were of the type normally used for engobe preparation: white clay, kaolin and bentonite.

It was found that all the studied engobe characteristics varied considerably on modifying the type of clayey material. The arising variations were shown to be due to changes in engobe plasticity and unfired layer packing on changing the type of clayey material.

Key words: Engobe, clayey materials, rheological behaviour, unfired layer properties

\section{INTRODUCCIÓN}

Los engobes se aplican sobre los soportes cerámicos con la finalidad de que actúen como interfase entre el soporte y el vidriado. Debido a ello, los engobes presentan composiciones de naturaleza intermedia entre las correspondientes a soportes y esmaltes: están formados tanto por materias primas plásticas (arcilla, caolín y bentonita) como no plásticas (frita, cuarzo, nefelina/feldespato, silicato de circonio, etc.) (1-2).

Las materias primas plásticas confieren a la composición de engobe un comportamiento reológico adecuado para efectuar su aplicación vía húmeda, mediante pistola o campana, y obtener una capa cruda con una resistencia mecánica y adherencia al soporte apropiadas para evitar la formación de grietas en dicha capa previamente a la cocción (3-4).

Cuando se aplica la suspensión de engobe sobre un soporte poroso se produce el paso del agua de la suspensión a la baldosa por el efecto de succión capilar generado por los poros de la pieza; a medida que la suspensión pierde agua se va for- mando una capa de sólido poroso a través de la cual deberá fluir el resto del agua de la suspensión hasta su completa absorción, por parte del soporte. Puede considerarse que el mecanismo por el que transcurre esta operación es análogo al de la circulación de un fluido en régimen laminar a través de un lecho poroso, descrito por Darcy (5). El comportamiento de la capa formada puede asimilarse, por su parte, al de un lecho formado por partículas esféricas, cuya constante de permeabilidad puede determinarse a partir de la ecuación:

$$
K p=\frac{d \cdot \varepsilon^{2}}{\pi \cdot 36 \cdot(1-\varepsilon)^{2}}
$$

donde

$d$ : diámetro medio de las partículas

$\varepsilon$ : porosidad total

$\pi$ : tortuosidad 
De esta forma, las características de esta capa que se va formando (porosidad y permeabilidad), las cuales dependen de la composición del engobe, influyen considerablemente no sólo sobre el tiempo necesario para que se endurezca el engobe aplicado (tiempo de secado superficial), sino también sobre el tiempo que tardarán en secarse las sucesivas aplicaciones posteriores. En este sentido, debe destacarse que, industrialmente, el tiempo de secado superficial se considera un factor crítico para la obtención de una baldosa cerámica esmaltada carente de defectos.

\section{OBJETO}

Además de las características reológicas de la suspensión, el tiempo de secado superficial (ts) y la compacidad y consistencia $(F)$ de la capa de engobe formada, afectan en gran medida al comportamiento del engobe en la operación de esmaltado. Por todo ello, se decidió estudiar en este primer trabajo, la influencia que ejerce la proporción de los diferentes componentes plásticos sobre el comportamiento reológico de la suspensión de engobe y sobre las características de la capa cruda de engobe formada.

\section{MATERIALES Y PROCEDIMIENTO EXPERIMENTAL}

\subsection{Materiales}

Las composiciones de los diferentes engobes se prepararon a partir de una frita, materias primas plásticas (arcilla, caolín y bentonita) y otras materias primas no plásticas (silicato de circonio, cuarzo y alúmina) de las habitualmente utilizadas para la preparación de este tipo de composiciones. En la tabla I se indica cada una de las composiciones ensayadas.

Tabla I

Composiciones de engobes

$\begin{array}{ccccccc}\text { Materias primas } & \text { C1 } & \text { C2 } & \text { C3 } & \text { C4 } & \text { C5 } & \text { C6 } \\ \text { Arcilla } & 20 & 15 & 10 & 15 & 10 & 15 \\ \text { Caolín } & - & - & - & 5 & 10 & - \\ \text { Bentonita } & - & - & - & - & - & 2 \\ \text { Frita } & 27 & 28.7 & 30.4 & 27 & 27 & 28 \\ \text { Silicato de } & 10 & 10.6 & 11.3 & 10 & 10 & 10.4 \\ \text { Circonio } & & & & & & \\ \text { Cuarzo } & 39 & 41.4 & 43.8 & 39 & 39 & 40.4 \\ \text { Alúmina } & 4 & 4.3 & 4.5 & 4 & 4 & 4.2\end{array}$

Ya que el trabajo consiste en determinar la influencia del tipo de materia prima plástica utilizada (arcilla, caolín y bentonita), a continuación se indican los resultados de la caracterización de las muestras, de cada uno de estos materiales, empleadas en la realización del trabajo. En la figura 1 se presentan las distribuciones granulométricas de la arcilla y el caolín, obtenidas por el método sedigraph. La distribución granulométrica de la bentonita no se pudo determinar debido a la dificultad que supone desleír dicha materia prima en agua. En la tabla II se detallan los valores de la superficie específica e índice de plasticidad, obtenidos por el método B.E.T. y por el método de indentación, respectivamente, correspondientes a todos los materiales plásticos utilizados.

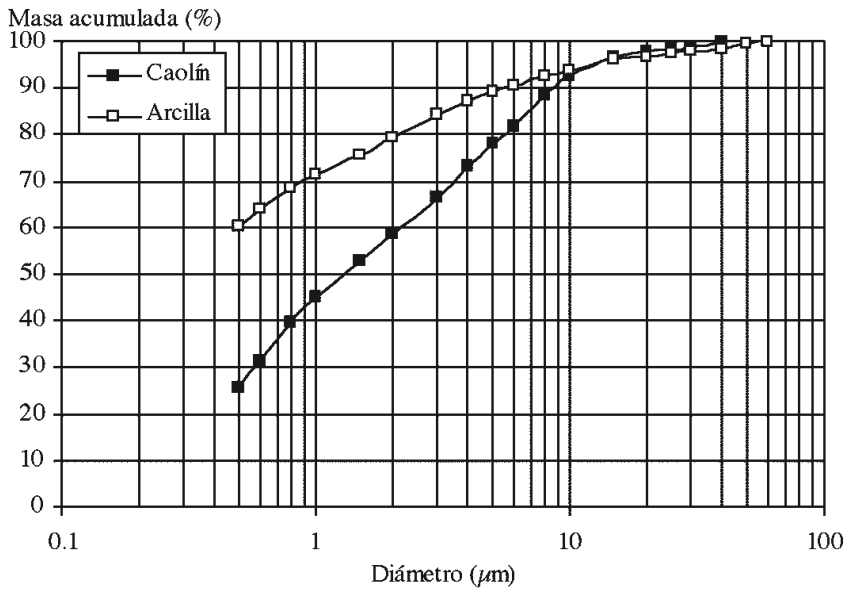

Figura 1. Distribución de tamaños de partícula de la arcilla y del caolín.

Tabla II

\begin{tabular}{|c|c|c|c|}
\hline & $\begin{array}{r}\text { Su} \\
\text { asticidad }\end{array}$ & $\begin{array}{l}\text { ica } \\
\text { les plást }\end{array}$ & \\
\hline & Arcilla & Caolín & Bentonit \\
\hline $\operatorname{Sp}\left(\mathrm{m}^{2} / \mathrm{g}\right)$ & 23.65 & 12.83 & 113.23 \\
\hline I.P (\%) & 31.1 & 22.1 & 289.3 \\
\hline
\end{tabular}

Como cabría esperar (6), la bentonita es la que mayor índice de platicidad presenta, debido a su elevada superficie específica, lo cual implica un pequeño tamaño de partícula, inferior al que presenta la arcilla. El caolín es el material arcilloso menos plástico de los ensayados, con una superficie específica inferior y un tamaño de partícula superior a los presentados por la arcilla.

\subsection{Procedimiento experimental}

\subsubsection{PREPARACIÓN DE LAS SUSPENSIONES DE ENGOBE}

Las suspensiones de engobe se prepararon molturando los diferentes componentes, en la proporción indicada en la tabla I, en un molino rápido de laboratorio con bolas de alúmina durante el tiempo necesario para alcanzar un rechazo aproximado del $1 \%$ en peso sobre tamiz de 40 micrómetros de luz de paso.

La molturación se realizó vía húmeda, siendo el contenido en sólidos empleado del $72 \%$ en peso, y preparándose todas las suspensiones en las condiciones correspondientes al mínimo de la curva de desfloculación. El desfloculante utilizado fue en todos los casos tripolifosfato sódico.

\subsubsection{CARACTERIZACIÓN DE LAS SUSPENSIONES DE ENGOBE}

A las suspensiones de engobe preparadas en la forma descrita se les determinó su comportamiento reológico mediante la realización de ensayos específicos para la obtención de la 
curva de flujo. Los ensayos se realizaron empleando un viscosímetro rotacional de cilindros concéntricos PHYSICA RHEOLAB MC-20, en el cual la muestra se mantenía a la temperatura de $25^{\circ} \mathrm{C}$. Así mismo, antes de proceder a la determinación propiamente dicha se sometió la muestra, durante cinco minutos, a una agitación intensa y, posteriormente, a seis minutos de reposo, con lo cual se evitaban los efectos debidos a la historia previa del material.

La curva de flujo se obtuvo controlando el gradiente de velocidad, el cual se aumentó progresivamente desde cero hasta un valor máximo de $1000 \mathrm{~s}^{-1}$ durante un tiempo de dos minutos. A continuación, la suspensión se mantuvo en agitación dos minutos al gradiente de velocidad máximo y, por último, se procedió a disminuirlo de forma controlada desde el valor máximo hasta el reposo, en un tiempo de dos minutos.

Para cada valor del gradiente de velocidad, el viscosímetro determina la fuerza de cizalla que ejerce sobre la muestra y calcula la viscosidad de la suspensión según la ley de Newton. De este modo se obtiene la variación de la fuerza de cizalla (curva de flujo) y de la viscosidad con el gradiente de velocidad (curva de viscosidad).

\subsubsection{CARACTERIZACIÓN DE LAS CAPAS CRUDAS DE ENGOBE}

\subsubsection{Determinación del tiempo de secado superficial}

Para la determinación del tiempo de secado superficial $\left(\mathrm{t}_{\mathrm{s}}\right)$ de la capa de engobe formada a partir de cada suspensión, se procedió a aplicar la suspensión mediante un recipiente que la contiene y que se desplaza, con la ayuda de un sistema automático, a una velocidad constante sobre el soporte a aplicar. En este caso se utilizó soporte poroso de revestimiento previamente cocido, y el espesor de la capa cruda aplicada era de, aproximadamente, $0.8 \mathrm{~mm}$. El tiempo de secado medido fue el transcurrido desde el momento de la aplicación hasta la pérdida de brillo de la superficie de la capa de engobe.

\subsubsection{Determinación de la compacidad}

La compacidad $(C)$ de un material conformado se define como el cociente entre el volumen que ocupa el sólido y el volumen total, siendo este último el formado por el sólido y los huecos (7).

$$
C=\frac{V s}{V t}=\frac{V s}{V s+V h}
$$

donde

$V s$ : Volumen de sólido

$V t$ : Volumen total

$V h$ : Volumen de huecos

Esta propiedad puede calcularse determinando la densidad real del sólido $\left(\rho_{s}\right)$ y la densidad aparente del material conformado $\left(\rho_{a p}\right)$, ya que:

$$
\rho_{s}=\frac{m s}{V s} \quad \rho_{a p}=\frac{m s}{V t}
$$

y sustituyendo en [2] se obtiene:

$$
C=\frac{\rho_{a p}}{\rho_{s}}
$$

La densidad real del sólido se determinó, mediante un picnómetro de helio, sobre una muestra del engobe en forma de polvo. Para ello, una porción de la suspensión de engobe se secó bajo lámparas de infrarrojos y con el polvo seco resultante se procedió a efectuar el ensayo.

La densidad aparente se determinó por inmersión en mercurio, sobre probetas conformadas por colado, las cuales habían sido obtenidas aplicando las suspensiones de engobe sobre soporte de revestimiento cocido, siendo el espesor de las mismas de, aproximadamente, $0.5 \mathrm{~cm}$.

\subsubsection{Determinación de la cohesión y adherencia}

Para determinar la cohesión de la capa consolidada, obtenida a partir de las suspensiones de engobe, y su adherencia al soporte, se empleó un aparato descrito con anterioridad en la bibliografía (4)(8). Este aparato consiste en una cuchilla unida, por un vástago, a la célula de carga de una máquina de ensayos universales que mide, de forma continua, la fuerza que es preciso aplicar para que penetre la cuchilla en el interior de la capa. El ensayo finaliza cuando se desprende o fractura la mencionada capa, anotando la fuerza máxima (F) que, hasta ese momento, ha sido registrada por la citada célula de carga.

\section{RESULTADOS Y DISCUSIÓN}

\subsection{Influencia del contenido en arcilla}

Para la realización de esta parte del trabajo se han preparado distintas composiciones de engobe a partir de una formulación estándar industrial (C1), reduciendo el porcentaje de arcilla y aumentando proporcionalmente el resto de materias primas, composiciones C2 y C3 indicadas en la tabla I.

Los resultados de la determinación del comportamiento reológico de las suspensiones, preparadas en la forma descrita en el apartado 3.2.1, se presentan en la figura 2. En ella puede observarse que, a medida que se reduce el contenido en mate-

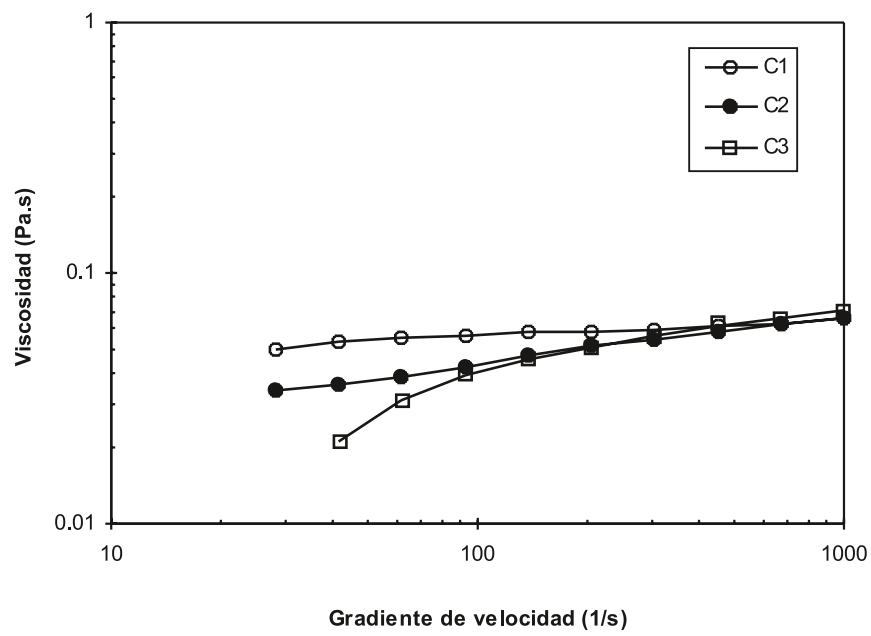

Figura 2. Influencia del contenido en arcilla sobre el comportamiento reológico de las suspensiones de engobe. 
rial plástico se acentúa el comportamiento dilatante de la suspensión debido a la disminución de la plasticidad de la composición de engobe (9).

En la tabla III se detallan los resultados de la caracterización de las capas crudas de engobe. En ella se observa que la disminución de la proporción de arcilla de un $20 \%$ a un $15 \%$, en la composición del engobe $\mathrm{C} 1$, no provoca una variación significativa en las propiedades de la capa obtenida a partir de dicho engobe, a excepción del valor de la cohesión y adherencia, el cual disminuye ligeramente. Cuando el porcentaje de arcilla en la composición C1 se disminuye hasta un $10 \%$, se puede observar que aunque la densidad aparente de la capa es prácticamente la misma (diferencias dentro del error de medida), la compacidad ha disminuido debido al aumento de la densidad del sólido. Asimismo, también disminuye el tiempo de secado superficial y el valor de la cohesión y adherencia de la capa consolidada.

Ello es posiblemente debido a que el empaquetamiento de las partículas va empeorando a medida que se eliminan del sistema las de pequeño tamaño que rellenaban inicialmente los huecos existentes. Este hecho produce un incremento en la porosidad de la capa $(\varepsilon)$, que provoca la disminución de su consistencia. Además, ello, unido al incremento del tamaño medio de partícula $(d)$, hace que, según la ecuación [1], aumenta la constante de permeabilidad $(K p)$ de la capa formada, de manera que el agua se elimina más fácilmente y se reduce el tiempo de secado superficial.

\begin{tabular}{|c|c|c|c|c|c|}
\hline \multicolumn{6}{|c|}{ Tabla III } \\
\hline Composición & ts (s) & $\mathbf{F}(\mathbf{N})$ & $\rho a p(g / c c)$ & $\rho s(\mathrm{~g} / \mathrm{cc})$ & Compacidad \\
\hline $\mathrm{C} 1$ & 47 & 117 & 2.004 & 2.89 & 0.693 \\
\hline $\mathrm{C} 2$ & 45 & 105 & 2.015 & 2.91 & 0.692 \\
\hline C3 & 40 & 100 & 2.009 & 2.93 & 0.686 \\
\hline $\mathrm{C} 4$ & 38 & 67 & 1.980 & 2.89 & 0.685 \\
\hline $\mathrm{C} 5$ & 24 & 70 & 1.936 & 2.89 & 0.670 \\
\hline $\mathrm{C} 6$ & 79 & 137 & 1.966 & 2.90 & 0.678 \\
\hline
\end{tabular}

\subsection{Influencia del contenido en caolín}

Para esta etapa del trabajo, se ha utilizado la formulación C1 modificando la proporción de caolín a costa del porcentaje de arcilla, composiciones C4 y C5 de la tabla I.

Del examen de las curvas de flujo y de viscosidad (figura 3)

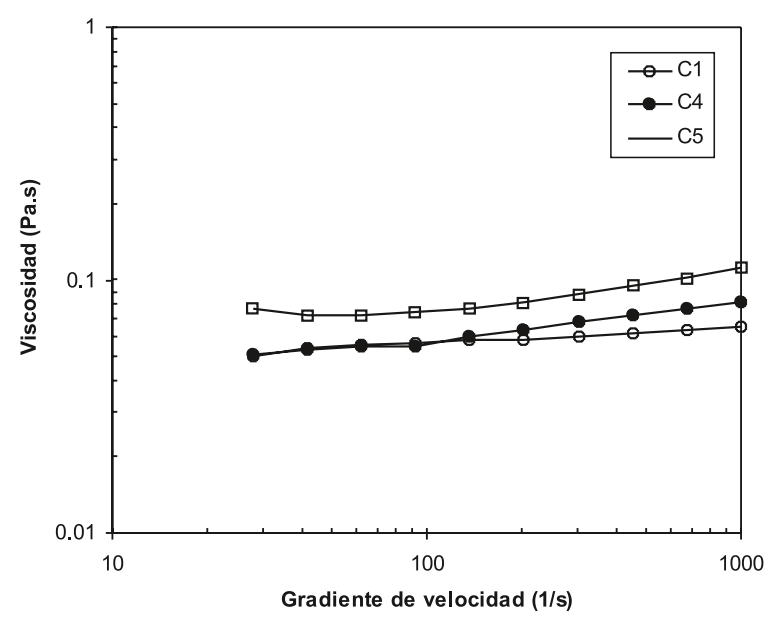

Figura 3. Influencia del contenido en caolín sobre el comportamiento reológico de las suspensiones de engobe. correspondientes a las composiciones $\mathrm{C} 1, \mathrm{C} 4$ y $\mathrm{C} 5$ se desprende que a medida que se incrementa el contenido en caolín, el moderado comportamiento dilatante de las suspensiones se hace más marcado, al tiempo que la viscosidad a elevadas velocidades de cizalla se hace mayor. Ello es debido a la reducción progresiva de la plasticidad de las suspensiones y al empeoramiento en el empaquetamiento de las partículas al eliminar una porción de material más fino (arcilla) y substituirlo por una más grueso (caolín) (9).

En la tabla III se indican las propiedades de las capas de engobe formadas. En ella se aprecia que al substituir arcilla por caolín en la composición C1 se produce un empeoramiento de las propiedades de la capa de engobe (menor tiempo de secado, menor adherencia y cohesión, y menor compacidad). Dicho empeoramiento en las propiedades puede explicarse teniendo en cuenta que al cambiar la arcilla por el caolín se está empeorando el empaquetamiento de las partículas (menor compacidad) dando lugar a capas más porosas, e incrementando el tamaño medio de aquellas, lo cual produce un aumento de la permeabilidad de la capa. Ello provoca el incremento de la velocidad de secado de esta, disminuyendo así el tiempo de secado superficial. Además, el hecho de que la capa formada sea más porosa repercutirá en una disminución de su consistencia (menores valores de F), dado que las fuerzas de unión entre las partículas son también menores.

\subsection{Influencia de la adición de bentonita}

La bentonita se emplea como aditivo para evitar los fenómenos de sedimentación en aquellas suspensiones que contienen un bajo porcentaje de material arcilloso (2). La razón de este comportamiento es la elevada superficie específica de las bentonitas, directamente relacionada con la elevada proporción de partículas de muy pequeño tamaño que presentan (6). Ambos hechos incrementan notablemente los contactos entre partículas y reducen la tendencia a la sedimentación. Dado el acusado efecto que provoca la adición de bentonita se procedió a introducir una pequeña cantidad de la misma en la composición de engobe C2, a costa de disminuir el porcentaje del resto de materias primas no plásticas, obteniéndose de esta forma la composición C6 (tabla I).

A la suspensión obtenida con esta composición se le determi-

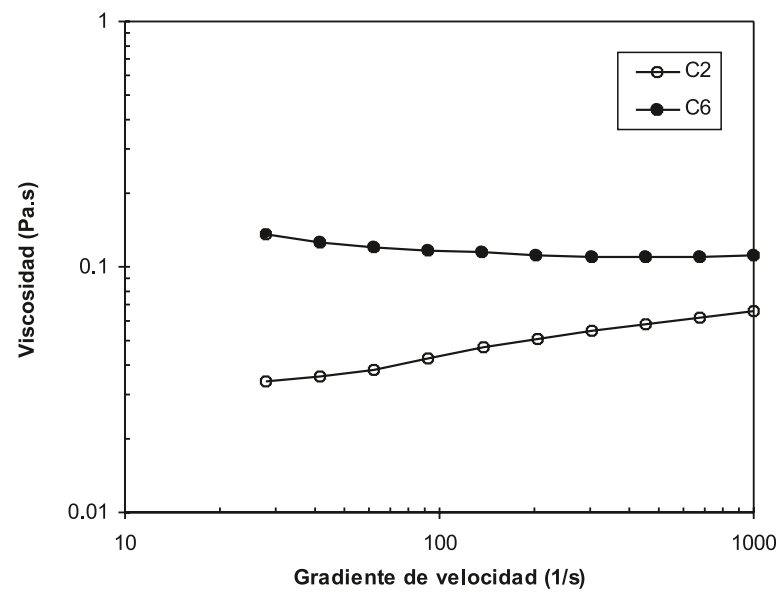

Figura 4. Influencia de la adición de bentonita sobre el comportamiento reológico de las suspensiones de engobe. 
nó el comportamiento reológico, obteniéndose los resultados que se indican en la figura 4 . Se incluye también en esta figura los resultados de la composición C2, a efectos comparativos.

En ella se observa que la adición de bentonita a la composición de engobe provoca un aumento acusado de la viscosidad, en todo el intervalo de gradientes de velocidad estudiado. Este efecto es debido al reducido tamaño de partícula que presenta la bentonita, ya que éstas tienen tendencia a formar estructura, la cual no se destruye con facilidad al someter la suspensión a una agitación (o gradiente de velocidad) determinada. Del mismo modo, el moderado comportamiento dilatante que presenta la composición C2 se convierte en un comportamiento más pseudoplástico, a causa de la elevada plasticidad que poseen este tipo de materiales.

En la tabla III se indican los resultados de la caracterización de las capas crudas de engobe. En ella puede observarse cómo la adición de bentonita a la composición de engobe provoca un aumento del tiempo de secado superficial y de la consistencia de la capa de engobe formada, mientras que la compacidad de la misma disminuye.

Así, al adicionar bentonita a la composición del engobe el empaquetamiento de las partículas presentes empeora, debido a la muy elevada proporción de partículas de pequeño y muy parecido tamaño que aporta aquella, lo cual provoca un aumento de la porosidad de la capa formada $(\varepsilon)$. Sin embargo, en este caso, ello no se traduce en una reducción de su consistencia y en un incremento de la velocidad de secado, sino justamente en lo contrario. Este hecho se debe probablemente a que, debido al pequeño tamaño de las partículas la tortuosidad $(\pi)$ de los capilares existentes en la capa formada es muy grande, lo que a la vista de la ecuación [1] se traduce en un aumento de la resistencia que opone el lecho de partículas al flujo de fluidos a su través, y por lo tanto, en una disminución de la velocidad de secado de la capa de engobe formada. Además, la capa formada a partir de la suspensión de engobe con bentonita resulta ser más consistente debido al aumento de las fuerzas de atracción de tipo electrostático entre las partículas de elevada superficie específica (6).

\section{CONCLUSIONES}

En el presente trabajo se ha estudiado la influencia que ejerce la proporción de cada uno de los materiales plásticos utilizados habitualmente en la composición de engobe sobre el comportamiento reológico de las suspensiones obtenidas a partir de estas composiciones, y sobre las características de la capa cruda formada a partir de ellas. Las conclusiones obtenidas son las siguientes:
Se ha demostrado que tanto las características reológicas de la suspensión como el tiempo de secado superficial, la compacidad y la consistencia de la capa cruda formada a partir de ella, dependen en gran medida de la naturaleza de los materiales plásticos presentes en la composición.

Una disminución de la proporción de arcilla blanca se traduce en un aumento del carácter dilatante de la suspensión y un empeoramiento de las propiedades de la capa cruda estudiadas (disminución de la compacidad, adherencia y tiempo de secado superficial), produciéndose ambos hechos como consecuencia de la disminución de la plasticidad del engobe.

La sustitución de la arcilla por caolín provoca un empeoramiento de todas las propiedades del engobe estudiadas, siendo de magnitud mayor que el observado al disminuir el porcentaje de arcilla. Este hecho es debido al carácter menos plástico del caolín con respecto a la arcilla.

La introducción de bentonita en la composición provoca un cambio muy acusado en el comportamiento reológico, haciéndolo más pseudoplástico, debido a la gran tendencia que tiene este material a formar estructura. Por otro lado, aunque la compacidad de la capa disminuye, el tiempo de secado superficial y la adherencia aumentan como consecuencia de la elevada plasticidad y superficie específica que presenta la bentonita.

\section{BIBLIOGRAFÍA}

1. C.W. Parmelee, Ceramic glazes. $3^{\text {rd }}$ ed. Boston: Cahners Books, 1973.

2. N. Tozzi, "Smalti Ceramici: considerazioni teoriche e pratiche". Faenza Editrice, 1992

3. A,Moreno, et al. "Comportamiento del engobe durante la operación de esmaltado". En: Oualicer 92: II Congreso Mundial de la Calidad del Azulejo y del Pavimento Cerámico. Castellón: Cámara Oficial de Comercio, Industria y Navegación, 1992, p. 395-412.

4. J. Bort,; J.L Fenollosa,. "Estudio de la adherencia en crudo de capas de esmalte". En: Qualicer 94: III Congreso Mundial de la Calidad del Azulejo y del Pavimento Cerámico. Castellón: Cámara Oficial de Comercio, Industria y Navegación, 1994, p. 93-109.

5. L. Svarovsky, (ed.). "Solid-liquid separation". London: Butterworths, 1977.

6. W.E Worall,. "Clays and ceramic raw materials". $2^{\text {nd }}$ ed. London: Elsevier,1986.

7. D.J. Cumberland,; R.J. Crawford, "The packing of particles". Amsterdam: Elsevier, 1987.

8. A. Belda,; et al. "Influencia del porcentaje de caolín, contenido en la capa de esmalte, sobre su comportamiento durante la cocción". En: Qualicer 98: V Congreso Mundial de la Calidad del Azulejo y del Pavimento Cerámico. Castellón: Cámara Oficial de Comercio, Industria y Navegación, 1998, p. 49-64

9. C.W. Macosko, Rheology. Principles, measuraments, and applications. New York: VCH Publishers, 1994.

Recibido: 11-6-99

Aceptado: 3-5-00 